\title{
Hypoxia and Cyanosis due to Unusual Right-to-Left Shunt in a Patient with an Atrial Septal Defect
}

Filipa Melao $^{1^{\star}}$, Cristina Cruz $^{1}$, Rafael Noya ${ }^{2}$, Joao Carlos Silva ${ }^{1}$ and Maria Júlia Maciel

${ }^{1}$ Serviço de Cardiologia, Centro Hospitalar Sao Joao, Porto, Portugal

${ }^{2}$ Serviço de Pneumologia, Centro Hospitalar Trás-os-Montes e Alto Douro, Vila Real, Portugal

*Corresponding author: Filipa Melao, Alameda Prof. Hernâni Monteiro, 4200 - 319 Porto, Portugal, E-mail: filipamelao@gmail.com

Received date: June 29, 2015; Accepted date: July 17, 2015; Published date: July 20, 2015

Copyright: (c) 2015 Melao F. This is an open-access article distributed under the terms of the Creative Commons Attribution License, which permits unrestricted use, distribution, and reproduction in any medium, provided the original author and source are credited.

\begin{abstract}
Hypoxia due to an Atrial Septal Defect (ASD), without Eisenmenger physiology, is reported rarely and may be underestimated due to difficulty in its diagnosis. We report the case of a 67-year-old woman with chronic cyanosis and hypoxia, in whom a large ASD was diagnosed by cardiac catheterization after an extensive workup to clarify the aetiology of the hypoxia. The right-to-left shunt at the ASD occurred probably due to a well-developed Eustachian valve, partly directing the right atrial flow into the left atrium, in the absence of pulmonary arterial hypertension.
\end{abstract}

Keywords: Cyanosis; Dyspnea; Heart septal defects

\section{Introduction}

Atrial Septal Defect (ASD) is the second most common congenital heart disease, accounting for 30 to $40 \%$ of congenital heart defects presenting in adulthood [1,2]. Ostium secundum type is the most frequent $(60-70 \%)$ [2]. When left untreated or undiagnosed these septal defects become symptomatic by the fifth decade of life, with dyspnoea on exertion and fatigue as the main presenting symptoms [1]. Chronic left-to-right shunting may lead to mild-to-moderate pulmonary arterial hypertension, and this in turn can cause shunt reversal, due to progressive increase of pulmonary vascular resistance, resulting in cyanosis, reduced exercise tolerance, development of right ventricular failure and premature death $[2,3]$. These septal defects are often identified incidentally (by echocardiography or autopsy due to the lack of early symptoms and the subtlety of physical findings), or in association with another cardiac abnormality, but hypoxemic ASD without significant pulmonary arterial hypertension are reported rarely and may be underestimated or undiagnosed $[3,4]$.

\section{Clinical case}

The authors describe the case of a 67-year-old caucasian female, retired (previous housemaid) with history of migraine and cyanosis since childhood, not investigated, that presented with a 2 -year complaint of moderate dyspnoea on exertion, associated with left chest pain. She had no dyspnoea at rest, orthopnoea, platypnoea, palpitations, cough, fever, hemoptysis, weight loss or anorexia. She never smoked nor had been chronically exposed to any toxic fumes or dusts. There was no family history of congenital heart disease or pulmonary disease. The physical exam revealed labial cyanosis, clubbing of the fingers and toes, and both lung and cardiac auscultation were normal. The jugular venous pulse and the carotid pulse were normal. There were no oedema of the legs, and the abdomen was soft and nontender, with no hepatosplenomegaly. She was evaluated by her primary physician, who requested a chest CT scan, which showed bilateral subpleural fibrosis and emphysema. Subsequently, she was oriented to a pneumology consultation for evaluation of chronic dyspnoea. An arterial blood gas was performed, which showed type I respiratory failure $(\mathrm{pH} \mathrm{7.38,} \mathrm{pO2} 55 \mathrm{mmHg}$, pCO2 $37 \mathrm{mmHg}$, oxygen saturation $88 \%$ on room air) and the patient was admitted to the hospital for study of hypoxia. The pulmonary function test was normal (FEV1/FVC 88; FVC 127; FEV 113; DLCO corrected 80.2). Laboratory examinations, which included complete blood count (haemoglobin $14.6 \mathrm{~g} / \mathrm{dL}$ and haematocrit $41.9 \%$ ); biochemistry (proBNP $58.84 \mathrm{pg} / \mathrm{mL}$ ), coagulation test, D-dimers $(0.22$ microg $/ \mathrm{mL}$ ), serology and autoimmune study were normal.

The spiral CT scan of the lungs showed a diffuse accentuation of peribronchovascular reticulum and subpleural densifications, raising the suspicion of an incipient idiopathic interstitial fibrosis or bronchiectasis. Also, a calcified granuloma was identified in the right upper lobe of the lung, without pleural or pericardial effusions. No signs of pulmonary embolism or neoformations were found. The ventilation/perfusion lung scan was normal.

The electrocardiogram showed sinus rhythm (heart rate $75 \mathrm{bpm}$ ) with left axis deviation. A transthoracic echocardiogram (limited by a poor acoustic window) revealed normal dimensions of ascending aorta and pulmonary artery; slight dilatation of the left atrium (area $22 \mathrm{~cm} 2$ ) with the remaining cardiac chambers within normal dimensions; normal thickness of the ventricular walls; pulmonary valve cusps were slightly thickened, with a mild pulmonary valve stenosis (maximal instantaneous gradient of $27 \mathrm{mmHg}$ ), mild to moderate mitral regurgitation, moderate tricuspid regurgitation (maximal instantaneous right ventricle/right atria gradient of $40 \mathrm{mmHg}$ ); left ventricular systolic function was preserved (ejection fraction 56\%); grade I diastolic dysfunction (E/A ratio $<1$ ) and normal right ventricular systolic function (TDI S' wave $17 \mathrm{~cm} / \mathrm{sec}$ ).

A six-minute walk test was performed to evaluate functional capacity, with the patient on supplemental oxygen therapy (FiO2 35\% by nasal cannula), stopped at 3:08 min due to oxygen desaturation of $24 \%$ (from $88 \%$ to $64 \%$ ) and she walked 240 meters (65\% of predicted) indicating a decreased exercise capacity.

Later, a cardiac MRI was performed, which documented a mild hypoplasia of the right ventricle and of the pulmonary trunk $(16 \mathrm{~mm}$ 
of diameter); trivial pulmonary valve stenosis (peak gradient $\sim 16$ $\mathrm{mmHg}$ ) with a proximal left pulmonary artery dilatation; hypertrabeculation of the left ventricle; inferior and inferolateral transmural fibrosis with ischemic pattern of the left ventricle; without evidence of intracardiac shunts (Qp:Qs 1:1); good ventricular systolic function (ejection fraction of the right and left ventricles were $74 \%$ and $61 \%$, respectively).

During hospitalization, the patient remained with sustained apyrexia but she always needed 3-4 L/min of oxygen supplement, by nasal cannula, to maintain oxygen saturation $>90 \%$ (stable hypoxia with a pO2 $\sim 60 \mathrm{mmHg}$, but although the oxygen saturation improved it remained $\sim 91 \%$ ), with a very good clinical tolerance.

Facing the hypothesis of a congenital heart malformation namely a pulmonary valve stenosis, the patient was referred to an Adult Congenital Heart Disease Clinic at a tertiary care hospital. She was discharged after one month of hospital admission, with the indication to maintain continuous oxygen supplement therapy $(4-6 \mathrm{~L} / \mathrm{min}$ by nasal cannula in order to maintain oxygen saturation $>90 \%)$. In the first appointment at the Adult Congenital Heart Disease Clinic, a transthoracic echocardiography was repeated, with the echocardiography findings similar to the previously described above, with no echocardiographic evidence of right chamber volume or pressure overload. Nevertheless, due to the poor acoustic window and to the extensive noninvasive imaging studies already performed on the patient, a cardiac catheterization was requested, after obtaining the patient's written consent, as an attempt to determine the degree and level of the pulmonary obstruction and to exclude an eventual anomalous systemic venous drainage to justify the chronic hypoxia with a normal Qp:Qs. This cardiac catheterization revealed a normal saturation in the pulmonary veins (inferior vena cava: $64.7 \%$; superior vena cava: 64.6\%; pulmonary veins: $98 \%$ ). Pressures and oxygen saturation of the remaining structures were as follows: $4 \mathrm{mmHg}$ in the right atrium, $24 / 8 \mathrm{mmHg}(67.9 \%$ oxygen saturation) in the main pulmonary artery, $6 \mathrm{mmHg}(91.8 \%)$ in the left atrium, and 152/80 $\mathrm{mmHg}(91 \%)$ in the aorta. A secundum ASD was angiographically detected with approximately $25 \mathrm{~mm}$ in diameter, which was occluded by a balloon catheter rising the systemic oxygen saturation to $100 \%$ (Figures 1 and 2); Qp:Qs=0.87. Coronary angiography showed no significant disease.

Subsequently, in order to better characterize the ASD, a transesophageal echocardiography was performed, which showed an ostium secundum ASD, measuring $20 \times 16 \mathrm{~mm}$; the Eustachian valve was slightly increased and targeted to the ASD (Figure 3). The pulmonary valve was thickened but with normal opening; the main pulmonary artery had $19 \mathrm{~mm}$ in diameter and the pulmonary veins drainage was normal.

Thus, the patient was proposed for percutaneous closure of the ostium secundum ASD, which was carried out with a $24 \mathrm{~mm}$ Figula device (the ASD diameter obtained by balloon measuring either by fluoroscopy or by echocardiography was $22 \mathrm{~mm}$ ), with a good final result and systemic saturation increased from 89 to $96 \%$ on room air.

After a 2 months follow-up, the patient is asymptomatic and with a good exercise capacity without oxygen supplement. She performed a control transthoracic echocardiography, 1 month after percutaneous ASD device closure that despite the poor acoustic window revealed a well deployed ASD device closure without apparent residual atrial shunt. Six months later, a follow-up transesophageal echocardiography showed no residual atrial shunt.

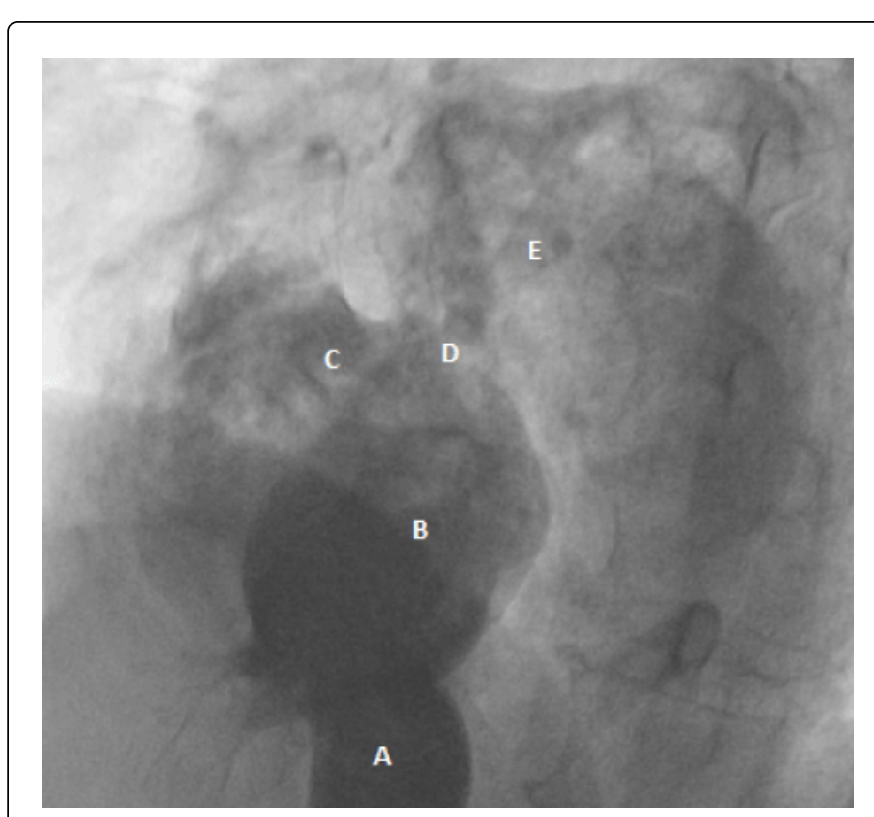

Figure 1: Cardiac catheterization showing passage of the contrast in the inferior vena cava (A) to the right atrium (B) and thus into the left atrium (D) via atrial septal defect (E). C - Superior vena cava.

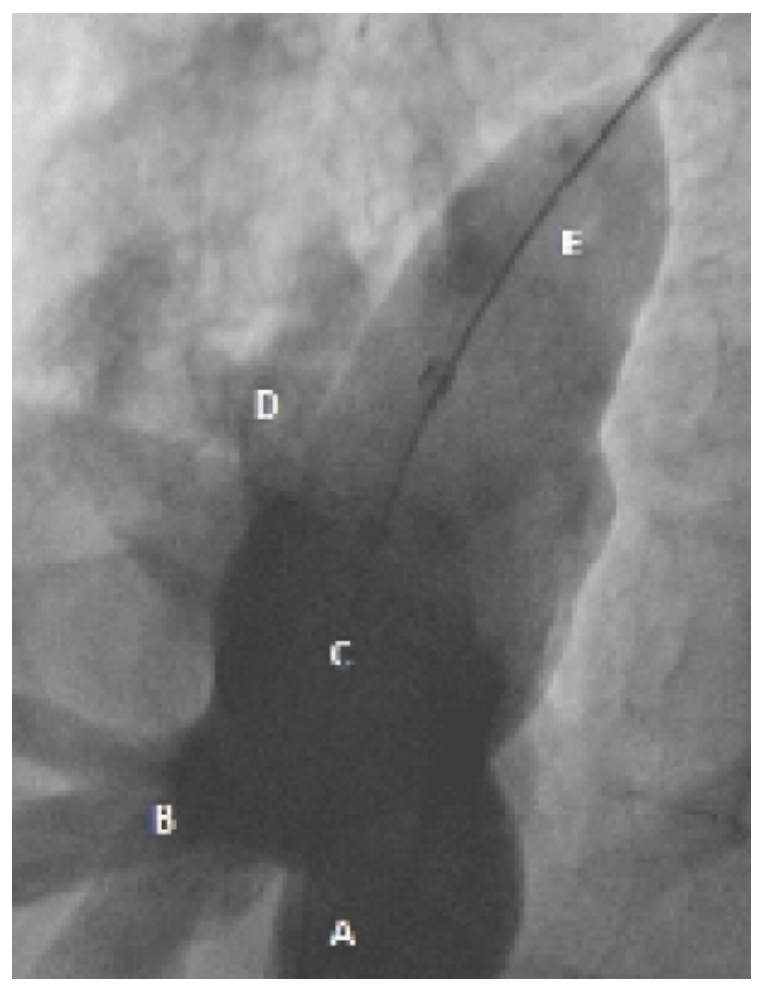

Figure 2: Cardiac catheterization showing occlusion of atrial septal defect by a balloon catheter (25mm in diameter). A - Inferior vena cava. B - Suprahepatic veins. C - Right atrium. D - Superior vena cava. E - Ballon catheter. 


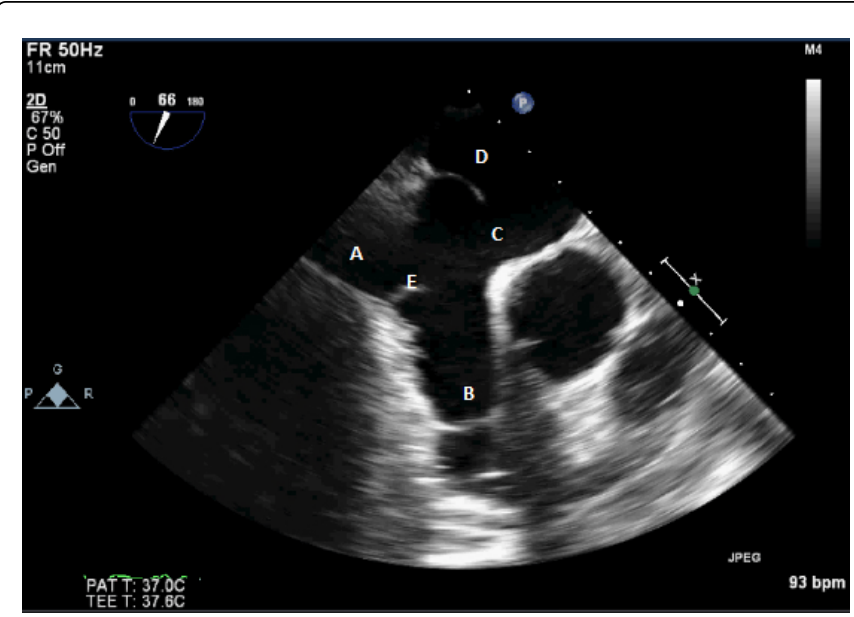

Figure 3: Transesophageal echocardiography revealed an ostium secundum atrial septal defect (C) and a slightly enlarged Eustachian valve (E) targeted to the atrial septal defect. A - Inferior vena cava. B - Right atrium. D - Left atrium.

\section{Discussion}

The magnitude of the atrial shunting depends on the compliance of the right and left ventricles, the systemic and pulmonary circulation resistance and size of the defect [1-4]. The typical feature of the ASD is the predominant left-to-right shunt, and subsequently over time, cyanosis and hypoxemia typically occurs when pulmonary arterial hypertension develops due to the progressive increase of pulmonary vascular resistance with shunt reversal [2-4].

Right-to-left shunt in the absence of elevated pulmonary vascular resistance is a rare condition which can also explain cyanosis and hypoxemia in the presence of an ASD $[1,2,4]$. Anatomical distortion of the heart can promote a right-to-left shunt due to an altered orientation of the inferior vena cava flow towards the atrial septum defect. This is commonly observed after pneumonectomy or dilatation/aneurism of the ascending aorta that modify the geometry or compresses the right atrium, respectively [3,4]. An exuberant Eustachian valve can exacerbate or cause such mechanism. Other causes are clinical conditions that may be associated with plathypnoeaorthodeoxia syndrome, pulmonary disease (pulmonary embolism, asthma, ARDS), chest trauma, tricuspid insufficiency and cardiac tamponade $[3,4]$. Other conditions with increased right-sided heart pressures (right ventricular infarction, atresia or stenosis of the pulmonary or tricuspid valve, anomalous venous return of vena cava, decreased diastolic compliance of right ventricle) may be involved [5]. In our patient the pulmonary arterial pressures and vascular resistance were normal. There were no significant malformations of the rightsided valves except for a slightly thickened pulmonary valve with a mild stenosis. The diastolic pressures of the right and left ventricles were similar. Both vena cava were normally connected to the right atrium. Furthermore, right ventricular hypoplasia was not sufficiently severe to be the cause of the right-to-left shunt, but led us to speculate that the preferential flow of the inferior vena cava into the left atrium, across the ASD by an overdeveloped Eustachian valve, could explain the right heart mild hypoplasia.

Transthoracic echocardiography remains the primary diagnostic modality for the detection of an ASD. Patients with inadequate acoustic imaging, questionable ASD, or unexplained RV volume overload, warrant further assessment with contrast echocardiogram and transesophageal echocardiography. Contrast echocardiography with agitated saline has a role in the detection of ASD with an important role in the evaluation of residual shunts after transcatheter closure [1].

In this case report, the only anatomical abnormal pattern we found was an overdeveloped Eustachian valve, which may justify cyanosis and hypoxia in the absence of pulmonary arterial hypertension, by the mechanism explained above.

\section{References}

1. Tobis J, Shenoda M (2012) Percutaneous treatment of patent foramen ovale and atrialseptal defects. J Am Coll Cardiol 60(18):1722-1732.

2. Krishnammoorthy S, Butt M, Lip GY (2010) Asymptomatic hypoxia in a young pregnant lady- unusual presentation of atrial septal defect. Int J Cardiol 143(2):e34-36.

3. El Tahlawi M, Jop B, Bonello B, Dragulescu A, Rouault F, et al. (2009) Should we close hypoxaemic patent foramen ovale and interatrial shunts on a systematic basis? Arch Cardiovasc Dis 102(11):755-759.

4. Solar M, Fridrich J, Ceral J, Borovec M (2007) Acute hypoxia due to right to left blood shunting in a patient with atrial septal defect. Resuscitation 74(2):382-385.

5. Ikäheimo MJ, Pokela RE, Kärkölä PJ, Takkunen JT (1983) Cyanotic ostium secundum atrial septal defect without pulmonary hypertension and clinical signs of heart disease-Report of two cases. Chest 84(5): 598-600. 